\title{
Associação entre ganho de peso gestacional e prognóstico da gestação
}

\author{
Association between gestational weight gain and pregnancy outcome \\ Danielle Góes da Silva ${ }^{1}$, Nayara Bispo Macedo ${ }^{2}$ \\ ${ }^{1}$ Professora Doutora do Departamento de Nutrição da Universidade Federal de Sergipe; ${ }^{2}$ Aluna do Curso de Nutrição da Universidade Federal de Sergipe.
}

Este estudo recebeu apoio financeiro por meio de bolsa concedida pelo Programa Especial de Inclusão em Iniciação Científica (PIIC) da Universidade Federal de Sergipe.

\section{RESUMO}

Objetivos: Avaliar associação entre ganho de peso gestacional e prognóstico da gestação.

Métodos: Um estudo transversal avaliou puérperas de uma maternidade do município de Aracaju, Sergipe, no período de março a junho de 2012. Os dados foram obtidos por meio de entrevistas, registros dos prontuários hospitalares e Cartão da Gestante. Foram pesquisadas variáveis relacionadas à gestação, condições do recém-nascido, retenção de peso no pós-parto e amamentação. Com base na classificação do ganho ponderal gestacional segundo as recomendações do Institute of Medicine, formaram-se três grupos: 1) grupo com ganho ponderal insuficiente; 2) grupo com ganho ponderal adequado; e 3) grupo com ganho excessivo. Na análise estatística foram utilizados os testes Qui-Quadrado e Análise de Variância, adotando nível de significância estatística inferior a 5\%.

Resultados: Foram incluídas no estudo 158 puérperas. Verificou-se alto percentual de mulheres que iniciaram a gestação com sobrepeso/ obesidade (30,4\%). Durante a gestação, não ocorreu ganho ponderal excessivo entre puérperas com baixo peso pré-gestacional, o que aconteceu em $25,2 \%$ das eutróficas, em $57,6 \%$ das com sobrepeso e em 53,3\% das obesas. O ganho ponderal excessivo durante a gestação foi associado com maior peso e comprimento do recém-nascido, maior prevalência de macrossomia fetal, maior índice de massa corporal materno e maior retenção de peso pela puérpera no terceiro mês pós-parto. Dificuldade em amamentar no terceiro mês pós-parto ocorreu em $4,5 \%$ das mães com ganho ponderal adequado durante a gestação e em 33,3\% daquelas com ganho de peso excessivo ( $\mathrm{p}=0,025)$. A ocorrência de parto cesáreo foi de $20 \%$ nas gestantes com ganho ponderal insuficiente, $40 \%$ nas que ganharam peso adequadamente e $47 \%$ naquelas com ganho ponderal excessivo $(\mathrm{p}=0,012)$.

Conclusões: Observaram-se valores maiores de ganho de peso excessivo entre mulheres que iniciaram a gestação com excesso de peso. O ganho de peso excessivo durante a gestação foi associado com maior peso médio e prevalência de macrossomia no recém-nascido, ocorrência de parto cesáreo, retenção de peso materno no pós-parto e dificuldade em amamentar.

DESCRITORES: GESTAÇÃO; SOBREPESO; OBESIDADE; GANHO DE PESO; RESULTADO DA GRAVIDEZ.

\section{ABSTRACT}

Aims: To evaluate the association between gestational weight gain and pregnancy outcome.

Methods: A cross-sectional study evaluated postpartum women from a maternity ward in a hospital located in Aracaju, Sergipe, Brazil, from March to June 2012. Data were obtained through interviews, medical records and records of prenatal care. Pregnancy-related variables, conditions of the newborn, weight retention in the postpartum period and breastfeeding were investigated. Based on the classification of gestational weight gain as recommended by the Institute of Medicine, three groups were formed: 1) group with insufficient weight gain; 2) group with adequate weight gain; and 3) group with excessive gain. Statistical analysis used chi-square and analysis of variance, with a level of statistical significance less than $5 \%$.

Results: At all 158 women were included in the study. There was a high percentage of women entering pregnancy with overweight/obesity (30.4\%), however, those with low pre-pregnancy weight did not gain excessive weight during their gestational period. Excessive gain was associated with greater weight and length of the newborn, higher prevalence of fetal macrosomy, increased maternal body mass index and higher postpartum weight retention in the third month after birth. Difficulty with breastfeeding in the third month postpartum occurred in $4.5 \%$ of mothers with adequate weight gain during pregnancy but it occurred in $33.3 \%$ of women with excessive weight gain $(p=0.025)$. A cesarean section was done in $20 \%$ of the women with insufficient gestational weight gain, in $40 \%$ of those who gained weight adequately and in $47 \%$ of those with excessive weight gain $(\mathrm{p}=0.012)$.

Conclusions: Excessive gestational weight gain was associated with higher average weight and prevalence of macrosomy in neonates, cesarean section, maternal weight retention in the postpartum and breastfeeding difficulty.

KEY WORDS: PREGNANCY; OVERWEIGHT; OBESITY; WEIGHT GAIN; PREGNANCY OUTCOME. 


\section{INTRODUÇÃO}

O ganho de peso durante o período gestacional mostra-se como variável determinante do resultado da gestação, tanto para a mãe quanto para o concepto. ${ }^{1-3} \mathrm{O}$ processo de transição nutricional vivenciado pela maioria das populações dos países em desenvolvimento tem resultado no aumento de mulheres com inadequação do estado nutricional tanto pré-gestacional quanto gestacional, devido à evolução insatisfatória do ganho ponderal. O fato de as mulheres hoje estarem em sua maioria com excesso de peso reflete-se em maior prevalência de mulheres que iniciam a gestação com estado nutricional inadequado e que continuam ganhando peso de forma excessiva durante a gestação. ${ }^{4}$

O ganho ponderal na gestação tem sido diretamente associado com o peso do recém-nascido. ${ }^{5,6}$ Há evidências de que, independente da raça e do estado nutricional pré-gestacional, o ganho ponderal excessivo durante a gravidez está associado a um risco elevado de neonatos grandes para idade gestacional. ${ }^{7}$ Além disso, o ganho ponderal da mãe durante a gestação pode agir como preditor da adiposidade total e distribuição de gordura corporal na infância ${ }^{8}$ e na adolescência, ${ }^{5}$ predispondo ao desenvolvimento de obesidade e doenças crônicas não transmissíveis, como diabetes e hipertensão arterial. ${ }^{4,9,10}$

Com relação à saúde materna, o excesso de ganho ponderal na gestação tem sido associado a intercorrências como diabetes gestacional, préeclâmpsia, parto cesáreo, infecções pós-parto e retenção do peso no período pós-parto. Um estudo mostrou que mulheres que ganharam peso dentro do recomendado tiveram quatro vezes mais chance de reter menos de 4,5 kg na sexta semana de pós-parto. ${ }^{11}$

Tendo em vista a importância do ganho de peso para o desfecho da gestação, na última década o Institute of Medicine (IOM), da National Academy of Sciences, Estados Unidos, com última atualização em 2009, propôs recomendações de ganho de peso adequado na gestação, com base no índice de massa corporal (IMC) pré-gestacional. ${ }^{12}$ Contudo, alguns estudos sugerem a revisão dessas recomendações no que diz respeito à elaboração de recomendações específicas para o grau de obesidade ${ }^{2}$ e a reavaliação dos pontos de corte, considerando não apenas os resultados do nascimento, mas também o risco de obesidade para a mãe e a criança. ${ }^{9}$

No Brasil, o Ministério da Saúde adotou a utilização da referência do $\operatorname{IOM}^{12}$ para avaliação e recomendação do ganho de peso na gestação; contudo, poucos estudos no país tem abordado a influência do ganho de peso da gestante sobre o desfecho da gestação. O presente estudo propõe-se a avaliar a associação entre o ganho de peso gestacional e o prognóstico da gestação.

\section{MÉTODOS}

Realizou-se um estudo prospectivo com puérperas de uma maternidade do município de Aracaju, capital do estado de Sergipe, no período de março a junho de 2012. Essa maternidade presta atendimento público e particular a municípios do estado de Sergipe e cidades circunvizinhas da Bahia e Alagoas, sendo realizados mensalmente cerca de 900 partos.

A seleção das participantes da pesquisa foi por conveniência (não aleatória), sendo incluídas puérperas que estavam presentes nos dias de coleta de dados e que possuíam registro das consultas do prénatal no Cartão da Gestante. Os critérios de exclusão foram: gestação múltipla, edema generalizado, idade abaixo de 20 anos e acima de 40 anos, baixa estatura (altura $<1,53 \mathrm{~m}$ ), desconhecimento do seu peso e da sua altura pré-gestacionais, desconhecimento da data da última menstruação e ausência de registro do peso a partir da $37^{\mathrm{a}}$ semana de gestação.

O projeto foi submetido e aprovado pelo Comitê de Ética da Universidade Federal de Sergipe com Certificado de Apresentação para Apreciação Ética $\mathrm{n}^{\circ}$ 00772612.7.0000.0058. As puérperas que concordaram em participar do estudo assinaram o Termo de Consentimento Livre e Esclarecido.

$\mathrm{Na}$ coleta de dados foram obtidas informações do Cartão da Gestante (idade, estatura, peso pré-gestacional, data da última menstruação, peso na última consulta pré-natal, paridade, diabetes, hipertensão arterial), do prontuário da maternidade (pressão sistólica e diastólica no parto, número de fetos, complicações durante a gravidez como pré-eclâmpsia diabetes gestacional), da Declaração de Nascidos Vivos (tipo de parto, semanas de gestação, peso, comprimento, perímetros cefálico e torácico do recémnascido, Apgar no $1^{\circ}$ e $5^{\circ}$ minutos). Foi feita uma entrevista no momento do pós-parto para obter informações sobre dificuldade no aleitamento materno e o tipo de aleitamento no primeiro dia pós-parto. No terceiro mês após o parto, foi feito contato telefônico para obter informações sobre peso e comprimento da criança, retenção de peso materno, tipo de aleitamento e dificuldades com a amamentação.

Com base no peso pré-gestacional e no peso do terceiro trimestre de gestação, foi calculado o 
ganho ponderal total durante a gestação. O peso prégestacional foi o peso antes de engravidar, obtido por meio do relato materno ou registro no Cartão da Gestante. Quanto ao peso no terceiro trimestre gestacional, foi considerado o peso da última consulta de pré-natal a partir da $37^{\mathrm{a}}$ semana de gestação, registrado no Cartão da Gestante.

As puérperas foram divididas em três grupos, de acordo com a recomendação de ganho de peso total na gestação proposta pelo $\mathrm{IOM}^{12} \mathrm{e}$ adotada pelo Ministério da Saúde no Brasil: A) Grupo com ganho insuficiente: mulheres de baixo peso com ganho de peso $<12,5 \mathrm{~kg}$, eutróficas com ganho $<11,5 \mathrm{~kg}$, mulheres com sobrepeso com ganho $<7 \mathrm{~kg}$ e obesas com ganho $<5 \mathrm{~kg}$; B) Grupo com ganho de peso adequado: mulheres de baixo peso com ganho entre 12,5-18 kg, eutróficas com ganho entre $11,5-16 \mathrm{~kg}$, mulheres com sobrepeso com ganho entre 7-11,5 kg e obesas com ganho entre 5-9 kg; e C) Grupo com ganho excessivo: mulheres de baixo peso com ganho de peso $>18 \mathrm{~kg}$, eutróficas com ganho $>16 \mathrm{~kg}$, mulheres com sobrepeso com ganho $>11,5 \mathrm{~kg}$ e obesas com ganho $>9 \mathrm{~kg}$. O estado nutricional prégestacional foi classificado com base nos pontos de corte de IMC da Organização Mundial de Saúde..$^{13} \mathrm{O}$ estado nutricional ao final da gestação foi avaliado com base na classificação do IMC por idade gestacional sugerida por Atalah et al. ${ }^{14}$

A tabulação dos dados e as análises estatísticas foram realizadas no programa SPSS, versão 18.0. Foi realizado o teste Qui-Quadrado para avaliar a associação da classificação do ganho de peso gestacional e estado nutricional pré-gestacional, além de características gestacionais, do parto e do pós-parto segundo ganho de peso gestacional. A análise de variância (ANOVA) foi utilizada para comparar as características gestacionais, do parto e do pós-parto entre os grupos com diferentes ganhos de peso. O nível de significância estatística adotado foi inferior a $5 \%$.

\section{RESULTADOS}

Foram avaliadas no total 214 puérperas. Destas, 56 foram excluídas do estudo por idade inferior a 20 anos $(n=6)$, idade maior que 40 anos $(n=1)$, baixa estatura $(n=7)$, desconhecimento do peso pré-gestacional $(n=2)$, ausência da informação do peso após a $37^{\mathrm{a}}$ semana de gestação $(n=31)$, desconhecimento da data da última menstruação $(n=4)$ e desconhecimento da estatura $(n=5)$. Dessa forma, a amostra incluiu 158 mulheres. A amostra de puérperas apresentou idade mediana de 27 anos (intervalo interquartil 23-31 anos, idade mínima 20 anos e máxima de 39 anos).
$\mathrm{Na}$ análise do estado nutricional pré-gestacional destaca-se o alto percentual de mulheres com excesso de peso (20,9\% sobrepeso e $9,5 \%$ obesidade, somando $30,4 \%$ ), ao passo que, no último trimestre de gestação, além dos altos percentuais de sobrepeso e obesidade, observam-se também mulheres com baixo peso (Figura 1).

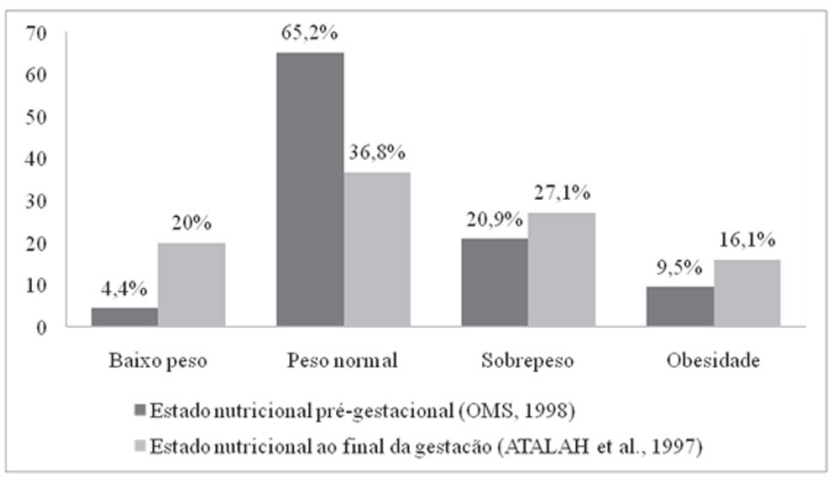

Figura 1. Estado nutricional pré-gestacional e ao final da gestação de uma amostra de 158 puérperas avaliadas em Aracaju, Sergipe, 2012.

Foi possível observar que entre as puérperas entrevistadas nenhuma tinha história de diabetes mellitus ou pressão arterial elevada antes da gestação. Uma gestante com estado nutricional pré-gestacional de sobrepeso teve diabetes gestacional e sete relataram pressão arterial elevada durante a gestação, sendo que duas destas eram eutróficas e cinco tinham excesso de peso.

Com relação à associação entre peso pré-gestacional e ganho de peso na gestação, observaram-se valores estatisticamente maiores de ganho de peso excessivo entre mulheres que iniciaram a gestação com excesso de peso. Ganho ponderal acima das recomendações do $\mathrm{IOM}^{12}$ não ocorreu entre puérperas com baixo peso pré-gestacional, ocorreu em $25,2 \%$ das eutróficas, em $57,6 \%$ das com sobrepeso e em $53,3 \%$ das obesas (Tabela 1).

As puérperas que ganharam peso insuficiente tiveram recém-nascidos com médias de peso ao nascer e comprimento inferiores quando comparadas às puérperas com ganho adequado e excessivo (Tabela 2). Além disso, o baixo peso ao nascer ocorreu na maioria dos recém-nascidos de mulheres com ganho insuficiente, enquanto que a macrossomia teve maior frequência em recém-nascidos de puérperas com ganho ponderal excessivo (Tabela 3). As gestantes com ganho ponderal insuficiente tiveram as maiores taxas de parto normal. Em comparação com as 
mulheres que ganharam peso insuficiente ou adequado durante a gestação, a ocorrência de parto cesáreo foi maior naquelas com ganho ponderal excessivo (Tabela 3).

No terceiro mês após o parto foi tentado contato telefônico com todas as mulheres, sendo obtida uma amostra de 78 mães, que corresponde a $49 \%$ da amostra inicial. Verificou-se que as mulheres que tiveram ganho ponderal acima do recomendado durante a gestação apresentavam maiores médias de IMC, sendo que a retenção de peso das mesmas foi de aproximadamente $6,5 \mathrm{~kg}$, enquanto mães que ganharam peso abaixo do normal retiveram menos de $0,5 \mathrm{~kg}$ e as que ganha- ram peso dentro do recomendado retiveram $2,7 \mathrm{~kg}$ (Tabela 2).

No terceiro mês após o parto, as mães que ganharam peso excessivo estavam em sua maioria com excesso de peso, ou seja, com sobrepeso e obesidade, sendo que as que ganharam peso insuficiente estavam em sua maioria em situação de baixo peso e eutrofia. Além disso, as mães com ganho excessivo relataram em maior número ter dificuldades em amamentar, com impressão de quantidade de leite insuficiente e problemas na amamentação, como pega incorreta e fissuras nos mamilos, quando comparadas às com ganho ponderal adequado (Tabela 3 ).

Tabela 1. Classificação do ganho de peso durante a gestação segundo estado nutricional pré-gestacional de uma amostra de 158 puérperas em Aracaju, Sergipe, 2012.

\begin{tabular}{|c|c|c|c|c|c|c|c|c|c|}
\hline \multirow{3}{*}{ Ganho ponderal na gestação ${ }^{1}$} & \multicolumn{8}{|c|}{ Estado nutricional pré-gestacional } & \multirow{3}{*}{$p$} \\
\hline & \multicolumn{2}{|c|}{$\begin{array}{l}\text { Baixo peso } \\
\quad(n=7)\end{array}$} & \multicolumn{2}{|c|}{$\begin{array}{l}\text { Eutrofia } \\
(n=103)\end{array}$} & \multicolumn{2}{|c|}{$\begin{array}{l}\text { Sobrepeso } \\
\quad(n=33)\end{array}$} & \multicolumn{2}{|c|}{$\begin{array}{l}\text { Obesidade } \\
\qquad(n=15)\end{array}$} & \\
\hline & $\mathbf{n}$ & $\%$ & $\mathbf{n}$ & $\%$ & $\mathbf{n}$ & $\%$ & $\mathbf{n}$ & $\%$ & \\
\hline Insuficiente & 4 & 57,1 & 36 & 35,0 & 5 & 15,2 & 5 & 33,3 & $0,078^{*}$ \\
\hline Adequado & 3 & 42,9 & 41 & 39,8 & 9 & 27,3 & 2 & 13,3 & $0,128+$ \\
\hline Excessivo & 0 & 0 & 26 & 25,2 & 19 & 57,6 & 8 & 53,3 & $0,000 \neq$ \\
\hline
\end{tabular}

${ }^{1}$ Segundo Instituto de Medicina. ${ }^{12}$

* Ganho de peso insuficiente versus Adequado e excessivo.

+ Ganho de peso adequado versus Insuficiente e excessivo.

₹ Ganho de peso excessivo versus Insuficiente e adequado.

Teste Qui-Quadrado.

Tabela 2. Características do recém-nascido e da mãe no parto e no terceiro mês pós-parto de acordo com o ganho de peso gestacional. Amostra de 158 puérperas, Aracaju, Sergipe, 2012.

\begin{tabular}{|c|c|c|c|c|c|c|c|}
\hline \multirow{2}{*}{ Características } & \multicolumn{2}{|c|}{ Ganho insuficiente } & \multicolumn{2}{|c|}{ Ganho adequado } & \multicolumn{2}{|c|}{ Ganho excessivo } & \multirow{2}{*}{$p^{*}$} \\
\hline & Média & DP & Média & DP & Média & DP & \\
\hline \multicolumn{8}{|l|}{ Recém-nascido } \\
\hline Idade gestacional (semanas) & 40,24 & 1,30 & 40,27 & 1,61 & 40,32 & 1,85 & 0,968 \\
\hline Peso ao nascer (g) & $3.235,8$ & 605,7 & $3.554,4$ & 433,4 & $3.611,3$ & 594,0 & 0,001† \\
\hline Comprimento ao nascer $(\mathrm{cm})$ & 48,31 & 3,54 & 49,84 & 1,67 & 50,01 & 2,26 & $0,002 \dagger$ \\
\hline Perímetro cefálico (cm) & 33,95 & 1,52 & 34,31 & 1,44 & 34,60 & 1,74 & 0,139 \\
\hline Perímetro torácico $(\mathrm{cm})$ & 33,22 & 1,81 & 33,71 & 1,60 & 33,73 & 2,17 & 0,336 \\
\hline Razão perímetro torácico e cefálico & 0,98 & 0,04 & 0,98 & 0,036 & 0,98 & 0,05 & 0,841 \\
\hline Índice de Rohrer & 3,01 & 1,57 & 2,86 & 0,27 & 2,89 & 0,26 & 0,708 \\
\hline Apgar $1^{\circ} \stackrel{\text { minuto }}{ }$ & 8,41 & 1,08 & 8,42 & 1,57 & 8,25 & 1,32 & 0,789 \\
\hline Apgar 5ㅇ minuto & 9,49 & 0,77 & 9,53 & 0,98 & 9,43 & 0,83 & 0,850 \\
\hline Peso no 3 o mês (g) & $6.442,2$ & 976,2 & $6.449,0$ & 866,4 & $6.806,9$ & $1.039,6$ & 0,315 \\
\hline Comprimento no $3^{\circ}$ mês $(\mathrm{cm})$ & 59,46 & 4,27 & 59,61 & 3,14 & 59,38 & 4,78 & 0,993 \\
\hline Ganho de peso no 3o mês (g) & $3.390,7$ & $1.162,5$ & $2.884,2$ & 722,2 & $3.012,6$ & $1.027,0$ & 0,190 \\
\hline \multicolumn{8}{|l|}{ Mãe } \\
\hline Pressão sistólica materna no parto (mmHg) & 118,14 & 10,75 & 119,6 & 12,57 & 124,13 & 13,26 & 0,057 \\
\hline Pressão diastólica materna no parto (mmHg) & 76,74 & 8,92 & 76,59 & 7,45 & 77,83 & 12,81 & 0,817 \\
\hline IMC no $3^{\circ}$ mês pós-parto $\left(\mathrm{kg} / \mathrm{m}^{2}\right)$ & 22,69 & 3,63 & 24,05 & 3,03 & 27,05 & 4,66 & $0,003+$ \\
\hline Retenção de peso no 3o mês pós-parto (kg) & 0,47 & 4,09 & 2,71 & 4,87 & 5,92 & 6,54 & $0,007+$ \\
\hline
\end{tabular}

DP = Desvio-padrão; IMC = Índice de Massa Corporal.

* ANOVA; + significativo $(<0,05)$. 
Tabela 3. Características gestacionais, do parto e do pós-parto segundo ganho de peso gestacional. Amostra de 158 puérperas, Aracaju, Sergipe, 2012.

\begin{tabular}{|c|c|c|c|c|c|c|c|}
\hline \multirow{2}{*}{ Características } & \multicolumn{2}{|c|}{ Ganho insuficiente } & \multicolumn{2}{|c|}{ Ganho adequado } & \multicolumn{2}{|c|}{ Ganho excessivo } & \multirow{2}{*}{ Valor $\boldsymbol{p}^{*}$} \\
\hline & n & $\%$ & $\mathbf{n}$ & $\%$ & n & $\%$ & \\
\hline Hipertensão arterial na gestação & & & & & & & 0,346 \\
\hline Sim & 2 & 4,0 & 1 & 1,8 & 4 & 7,5 & \\
\hline Não & 48 & 96,0 & 54 & 98,2 & 49 & 92,5 & \\
\hline Parto prematuro & & & & & & & 0,849 \\
\hline Sim & 1 & 2,0 & 2 & 3,64 & 2 & 3,8 & \\
\hline Não & 49 & 98,0 & 53 & 96,36 & 51 & 96,2 & \\
\hline Tipo de parto & & & & & & & 0,012 \\
\hline Normal & 40 & 80,0 & 33 & 60,0 & 28 & 52,8 & \\
\hline Cesárea & 10 & 20,0 & 22 & 40,0 & 25 & 47,2 & \\
\hline Peso ao nascer & & & & & & & 0,044 \\
\hline Baixo peso $(<2.500 \mathrm{~g})$ & 2 & 4,0 & 0 & 0,0 & 1 & 1,9 & \\
\hline Peso adequado (2.500g |- 4.000g) & 45 & 90,0 & 44 & 80,0 & 37 & 71,2 & \\
\hline Macrossomia ( $\geq 4.000 \mathrm{~g})$ & 3 & 6,0 & 11 & 20,0 & 14 & 26,9 & \\
\hline Dificuldade em amamentar no $1^{\circ}$ dia pós-parto & & & & & & & 0,938 \\
\hline Sim & 11 & 22,0 & 13 & 23,6 & 13 & 25,0 & \\
\hline Não & 39 & 78,0 & 42 & 76,4 & 39 & 75,0 & \\
\hline Tipo de aleitamento no $1^{\circ}$ dia pós-parto & & & & & & & 0,551 \\
\hline Aleitamento materno exclusivo & 35 & 70,0 & 38 & 69,1 & 31 & 59,6 & \\
\hline Aleitamento misto & 12 & 24,0 & 10 & 18,2 & 14 & 29,6 & \\
\hline Aleitamento artificial & 3 & 6,0 & 7 & 12,7 & 7 & 13,5 & \\
\hline IMC materno no 3 o mês pós-parto & & & & & & & 0,017 \\
\hline Baixo peso e Eutrofia & 17 & 77,3 & 9 & 64,3 & 6 & 33,3 & \\
\hline Sobrepeso e Obesidade & 5 & 22,7 & 5 & 35,7 & 12 & 66,7 & \\
\hline Tipo de aleitamento no $3^{\circ}$ mês pós-parto & & & & & & & 0,308 \\
\hline Aleitamento materno exclusivo & 14 & 50,0 & 12 & 57,1 & 13 & 50,0 & \\
\hline Aleitamento misto & 10 & 35,7 & 8 & 38,1 & 6 & 23,1 & \\
\hline Aleitamento artificial & 4 & 14,3 & 1 & 4,8 & 7 & 26,9 & \\
\hline Dificuldade em amamentar no $3^{\circ}$ mês pós-parto & & & & & & & 0,025 \\
\hline Sim & 4 & 13,8 & 1 & 4,5 & 9 & 33,3 & \\
\hline Não & 25 & 86,2 & 21 & 95,5 & 18 & 66,7 & \\
\hline
\end{tabular}

IMC = Índice de Massa Corporal.

* Teste Qui-Quadrado.

\section{DISCUSSÃO}

O presente estudo constatou um alto percentual de mulheres que iniciou a gestação com excesso de peso e ganhou peso de forma inadequada durante a gestação. Percebe-se que o estado nutricional das gestantes tende a piorar ao final da gestação e este achado é preocupante, pois há evidências de que existem determinadas fases da vida onde há uma maior vulnerabilidade à obesidade, e entre elas estão os períodos de ganho de peso gestacional e amamentação. ${ }^{15}$ Sabe-se que a quantidade de mulheres em idade reprodutiva com sobrepeso tem aumentado, juntamente com a epidemia mundial de obesidade. ${ }^{4}$

Este estudo identificou que mais da metade das mulheres estudadas tiveram ganho ponderal inadequado na gestação, considerando as recomendações do IOM. ${ }^{12}$ Apesar de serem classificações de IMC diferentes, observam-se alterações significativas no estado nutricional das puérperas, já que houve um aumento da quantidade de mulheres com baixo peso, sobrepeso e obesidade no período gestacional, cabendo chamar atenção ao alto percentual de mulheres com excesso de peso (sobrepeso e obesidade). 
Estudo realizado por Marano et al. ${ }^{16}$ com mulheres no primeiro trimestre de gestação dos municípios de Petrópolis e Queimados (estado do Rio de Janeiro) observou inadequação no ganho de peso gestacional em $71,4 \%$ das gestantes, sendo que, $35,6 \%$ ganharam peso insuficiente e $35,8 \%$ acima do recomendado, resultado semelhante ao do atual estudo. Mulheres com sobrepeso e obesidade tiveram maior chance de ganhar peso acima do recomendado em um estudo realizado por Fazio et al., ${ }^{17}$ onde o ganho ponderal acima das recomendações do IOM ocorreu em 17,4\% das gestantes com baixo peso, 31,0\% das eutróficas, $56,7 \%$ das com sobrepeso e $39,5 \%$ das obesas. Esses dados vêm ao encontro dos achados deste estudo, onde se percebeu que as gestantes que iniciam a gestação em situação nutricional de risco tendem a piorá-lo com o andamento da gestação.

Estudo feito por Park et al., ${ }^{18}$ que investigou a associação entre as recomendações de ganho de peso do IOM e resultados adversos no recém-nascido, mostrou que havia maior proporção de neonatos grandes para a idade gestacional entre mulheres que tiveram excesso de ganho de peso gestacional, além do que, o ganho de peso gestacional maior que o recomendado pelo IOM estava associado com menores chances de neonatos pequenos para idade gestacional. Também no presente estudo gestantes com ganho de peso insuficiente na gestação tiveram recém-nascidos com menores pesos e comprimentos ao nascer, ao contrário daquelas com ganho ponderal excessivo, que tiveram recém-nascidos maiores. Em outro estudo, conduzido por Crane et al., ${ }^{19}$ mulheres que tiveram ganho ponderal excessivo na gestação, incluindo as eutróficas, as com sobrepeso, as obesas e as obesas mórbidas, tinham mais chance de gerarem neonatos macrossômicos. Estudo feito por Langford et al., ${ }^{1}$ que analisou a associação entre ganho de peso gestacional e resultados adversos para a mãe e o concepto em mulheres com sobrepeso, mostrou que nas mesmas o ganho de peso gestacional acima do recomendado pelo IOM foi diretamente associado ao risco de macrossomia fetal. Também no estudo de Langford et al., ${ }^{1}$ mulheres com sobrepeso que ganharam peso gestacional acima do recomendado pelo IOM tiveram maior risco de parto cesárea, concordando com o resultado obtido no presente estudo.

O excesso de peso na gestação tem sido associado a prejuízos nas condições de aleitamento. ${ }^{20,21}$ Um estudo mostrou que gestantes obesas tiveram menor chance de manter o aleitamento materno exclusivo, recorrendo ao uso de fórmulas. ${ }^{22}$ Além disso, a revisão de literatura feita por Wojcicki ${ }^{23}$ mostrou que o início e a duração do aleitamento podem ser tanto mais prejudicados quanto maior for o IMC materno, fatos confirmados pelos resultados obtidos neste estudo. Outra revisão de literatura mostrou que o excesso de adiposidade materna pode influir no desenvolvimento das glândulas mamárias antes da concepção, durante a gravidez e durante a lactação, porém estudos sobre os impactos do ganho ponderal da gestante no aleitamento ainda são limitados. ${ }^{24}$

O segundo contato com as mães no terceiro mês após o parto teve como objetivo investigar as condições de aleitamento e a retenção de peso materno, assim como no estudo de Marano et al., ${ }^{16}$ feito em diferentes etapas (gestação, pós-parto, três e seis meses após o parto). Cabe destacar a associação entre ganho de peso gestacional excessivo e aumento de IMC no terceiro mês pós-parto, retenção de peso pós-parto e prevalência de excesso de peso entre as mães. Revisão de literatura realizada por Castro et al. ${ }^{25}$ apontou que as principais causas da alteração de peso no período pós-parto são ganho de peso gestacional acima das recomendações do IOM, IMC pré-gestacional $\geq 25 \mathrm{~kg} / \mathrm{m}^{2}$, dieta, tempo e intensidade do aleitamento materno e fatores sociodemográficos, como etnia negra, primiparidade, idade materna alta, baixa renda e baixa escolaridade. Além disso, comenta que as ações de saúde costumam priorizar a garantia do aleitamento em detrimento da perda de peso materna durante esse período e que a prática regular de atividade física serviria como modo de evitar o ganho de peso gestacional excessivo e acelerar a perda de peso no pós-parto. ${ }^{25}$

Cohen e Koski ${ }^{11}$ investigaram se a retenção de peso pós-parto seria minimizada por variáveis como seguir a recomendação de ganho de peso baseada no IMC prégestacional e/ou alcançar o ganho ponderal semanal ou total recomendado, mostrando que mulheres que ganharam peso semanal dentro do recomendado tiveram quatro vezes mais chance de reter menos de 4,5 $\mathrm{kg}$ na sexta semana pós-parto. Esses autores comentam que ganho de peso adequado, consumo menos calórico e atividade física deveriam ser enfatizados em programas educacionais de pré-natal. ${ }^{11}$

O acompanhamento e orientações nutricionais durante esse período que favorece as alterações no estado nutricional são de suma importância para melhorar o prognóstico gestacional. Vitolo et al. ${ }^{26}$ verificaram que orientações alimentares tiveram resultados positivos em diminuir o ganho de peso de gestantes com excesso de peso e em reduzir condições como diabetes gestacional, pré-eclâmpsia, baixo peso e prematuridade.

Apesar dos relevantes achados, este estudo apresenta limitações, como os possíveis erros relacionados 
à forma de obtenção dos pesos (materno e do neonato), realizada a partir dos registros (Cartão da Gestante ou prontuários) e do autorrelato materno. Além disso, cabe destacar a perda amostral de cerca de metade da amostra inicial na fase de contato telefônico com as mães, no terceiro mês pós-parto.

Em conclusão, observaram-se altos percentuais de ganho de peso gestacional inadequado entre as mulheres avaliadas, sendo que o ganho ponderal gestacional excessivo foi associado com maior prevalência de macrossomia fetal, cesariana, maior retenção de peso materno no pós-parto e dificuldade em amamentar. Destaca-se, assim, a importância do acompanhamento nutricional durante a gestação, especialmente de mulheres com excesso de peso, a fim de minimizar possíveis complicações gestacionais, e a necessidade de monitoramento da retenção de peso no período pós-parto, a fim de favorecer uma adequada recuperação do estado nutricional.

\section{AGRADECIMENTOS} de bolsa.

Ao Programa Especial de Inclusão em Iniciação Científica (PIIC) da Universidade Federal de Sergipe, pela concessão

\section{REFERÊNCIAS}

1. Langford A, Joshu C, Chang JJ, Myles T, Leet T. Does gestational weight gain affect the risk of adverse maternal and infant outcomes in overweight woman? Matern Child Health J. 2011;15(7):860-5.

2. Blomberg M. Maternal and neonatal outcomes among obese women with weight gain below the new institute of medicine recommendations. Obstet Gynecol. 2011;117(5):1065-70.

3. Olson G, Blackwell SC. Optimization of gestational weight gain in the obese gravida: a review. Obstet Gynecol Clin North Am. 2011;38(2):397-407.

4. Mattar R, Torloni MR, Betrán AP, Merialdi M. Obesidade e gravidez. Rev Bras Ginecol Obstet. 2009; 31(3):107-10.

5. Wrotniak BH, Shults J, Butts S, Stettler N. Gestational weight gain and risk of overweight in the offspring at age 7y in a multicenter, multiethnic cohort study. Am J Clin Nutr. 2008;87(6):1818-24.

6. Ludwig DS, Currie J. The association between pregnancy weight gain and birthweight: a within-family comparison. Lancet. 2010;376(9745):984-90.

7. Bowers K, Laughon SK, Kiely M, Brite J, Chen Z, Zhang C. Gestational diabetes, pre-pregnancy obesity and pregnancy weight gain in relation to excess fetal growth: variations by race/ethnicity. Diabetologia. 2013;56(6):1263-71.

8. Dello Russo M, Ahrens W, De Vriendt T, Marild S, Molnar D, Moreno LA, Reeske A, Veidebaum T, Kourides YA, Barba G, Siani A; IDEFICS Consortium. Gestational weight gain and adiposity, fat distribution, metabolic profile, and blood pressure in offspring: the IDEFICS project. Int J Obes (Lond). 2013;37(7):914-9. doi: 10.1038/ijo.2013.35.

9. Oken E, Taveras EM, Kleinman KP, Rich-edwards JW, Gillman MW. Gestational weight gain and child adiposity at age 3 years. Am J Obstet Gynecol. 2007;196(4):322.

10. Oken E, Rifas-Shiman SL, Field AE, Frazier AL, Gillman MW. Maternal gestational weight gain and offspring weight in adolescence Obstet Gynecol. 2008;112(5):999-1006.

11. Cohen TR, Koski KG. Limiting excess weight gain in healthy pregnant women: importance of energy intakes, physical activity, and adherence to gestational weight gain guidelines. J Pregnancy. 2013;2013:787032. doi: 10.1155/2013/787032.

12. Institute of Medicine, National Academy of Sciences. Weight gain during pregnancy: reexamining the guidelines. Washington (DC): National Academy Press; 2009. [cited 2014 Mar 12]. Available from: http://www.iom.edu/ /media/Files/Report\%20Files/2009/WeightGain-During-Pregnancy-Reexamining-the-Guidelines/Report\%20Brief\%20-\%20Weight\%20Gain\%20During\%20Pregnancy.pdf

13. World Health Organization. Division of Noncommunicable Diseases. Programme of Nutrition Family and Reproductive Health. Obesity: preventing and managing the global epidemic: report of a WHO consultation on obesity. Geneva: WHO; 1998. [cited 2013 Dec 16]. Available from: http://whqlibdoc.who.int/hq/1998/WHO_NUT_NCD_98.1_(p1-158).pdf

14. Atalah SE, Castillo CL, Castro RS, Aldea PA. Propuesta de um nuevo estandar de evaluación nutricional en embarazadas. Rev Med Chile. 1997;125(12):1429-36.

15. Drehmer M. Ganho de peso gestacional, desfechos adversos da gravidez e retenção de peso pós-parto [dissertation]. [Porto Alegre]: Universidade Federal do Rio Grande do Sul; 2010. 233p.

16. Marano D, Gama SGN, Pereira APE, Souza Junior PRBJ. Adequação do ganho ponderal de gestantes em dois municípios do estado do Rio de Janeiro (RJ), Brasil, 2008. Rev Bras Ginecol Obstet. 2012;34(8):386-93.

17. Fazio ES, Nomura RMY, Dias MCG, Zugaib M. Consumo dietético de gestantes e ganho ponderal materno após aconselhamento nutricional Rev Bras Ginecol Obstet. 2011;33(3):87-92.

18. Park S, Sappenfield WM, Bish C, Salihu H, Goodman D, Bensyl DM. Assessment of the Institute of Medicine recommendations for weight gain during pregnancy: Florida, 2004-2007. Matern Child Health J. 2011;15(3):289-301. 
19. Crane JM, White J, Murphy P, Burrage L, Hutchens D. The effect of gestational weight gain by body mass index on maternal and neonatal outcomes. J Obstet Gynaecol Can. 2009;31(1):28-35.

20. Rasmussen KM, Kjolhede CL. Prepregnant overweight and obesity diminish the prolactin response to suckling in the first week postpartum. Pediatrics. 2004;113(5):e465-71.

21. Liu J, Smith MG, Dobre MA, Ferguson JE. Maternal obesity and breast-feeding practices among white and black women. Obesity (Silver Spring). 2010;18(1):175-82.

22. Ayerza Casas A1, Rodríguez Martínez G, Samper Villagrasa MP, Murillo Arnal P, Alvarez Sauras ML, Moreno Aznar LA, Olivares López JL; Grupo Colaborativo CALINA. Características nutricionales de los recién nacidos de madres con sobrepeso y obesidad. An Pediatr (Barc). 2011;75(3):175-81.

23. Wojcicki JM. Maternal prepregnancy body mass index and initiation and duration of breastfeeding: a review of the literature. J Womens Health. 2011;20(3):341-7.

24. Rasmussen KM. Association of maternal obesity before conception with poor lactation performance. Annu Rev Nutr. 2007; 27:103-21.

25. Castro MBT, Kac G, Sichieri R. Determinantes nutricionais e sóciodemográficos da variação de peso no pós-parto: uma revisão da literatura. Rev Bras Saúde Mater Infant. 2009;9(2):125-37.

26. Vitolo MR, Bueno MSF, Gama CM. Impacto de um programa de orientação dietética sobre a velocidade de ganho de peso de gestantes atendidas em unidades de saúde. Rev Bras Ginecol Obstet. 2011;33(1):13-9. 